\title{
Numerical Solutions for Transverse Loading on Plate made of Functionally Graded Material
}

\author{
Manish Bhandari \\ Assistant Professor, Department of Mechanical Engineering, JNV University, Jodhpur, (Rajasthan), India
}

\begin{abstract}
The Functionally gradient materials (FGM) are developed by looking into the concept of gradual variation of material properties as compared to composites so as to meet various engineering applications where high thermal resistance and mechanical properties are in demand such as nuclear sector, aerospace sector etc. Since the FGMs are in place of homogeneous materials, it is pertinent to exhibit the comparison of the characteristics of FGMs and homogeneous materials. These studies are concerned with stress, deformation and stability problems of FGM plates accounting for various effects, such as geometric and physical non-linearity and transverse shear deformability. In the present research, an FGM plate is considered of which the properties are varies considering power law, sigmoid law and exponential law. The plate is applied with uniformly distributed load and point load, the response is recorded with varying aspect ratio and variation in volume fraction index. The results are exhibited in terms of on dimensional parameters such as non dimensional deflection, tensile stress, shear stress, strain and shear strain.
\end{abstract}

Key words: Functional composites, Elastic properties, Finite element analysis (FEA).

\section{INTRODUCTION}

The property of the FGM can be utilized to accomplish the needs of various engineering sectors to achieve the advantage of the properties of factor materials. It is possible because of the material composition of the FGM which varies as per certain laws in a preferred direction. Hence the deformation behaviour of FGM structures have attracted attention of engineering in the past few years which contains aerospace structures, heat engine components and nuclear power plants etc. Since the FGMs are used in place of homogeneous materials, the assessment of static, dynamic and mechanical deformation behaviour of functionally graded plate structures depends on the plate model kinematics and modelling techniques. These studies are related with stress, deformation and stability problems of FGM structures. Finite element analysis of transient response of FG cylinders and plates have been formulated theoretically and conducted considering nonlinearity based on the higher order or third-order shear deformation theory of plates [1].Quasi-static experiments have been conducted for crack of FGM and the stress intensity factors have been found out [2].Modeling of Functionally Graded Materials by Numerical Homogenization have been performed and the accuracy of the method is satisfactory [3].The results of P-FGM, S-FGM and E-FGM are evaluated by the numerical solutions (FEA) from theoretical formulations[4].Analytical method to analyze displacements and stresses in a functionally graded composite beam subjected to transverse load are developed and the results obtained from this method were compared with the finite element solution done by ANSYS [5]. A second-order homogenization method is presented for structure made of FGM [6]. Static analysis of an isotropic rectangular plate with some boundary conditions and various load applications have been shown [7]. A review is done of research on FG plates and thrown light on the important research work [8]. Elasticity solutions for FG rectangular plates with two opposite edges simply supported have been presented along with the application of the solutions [9]. FGM plate as structural member is subjected to various loading conditions and the behaviour of the plate is presented [10]. FEM model based on a triangular flat shell element, with 3 nodes and 8 DOF per node, associated with a HSDT is used to model FGM plate [11]. Latest software techniques have been used by researchers to detect the behavior of the FGMs. [12-14]. The present paper the ratio of side lengths of FGM plate is varied and transverse load is applied in form of udl and point load. The plate is simulated and various geometric parameters such as deflection, stress and strain are computed for P-FGM, S-FGM and E-FGM. The finite element method and simulation software are used for computational analysis.

\section{METHODOLOGY \\ 2.1 Material}

Ceramic and metal are used for the graded materials. The metal is Aluminum (Al) and the ceramic is Zirconia ( $\mathrm{ZrO} 2)$. The properties such as Young's modulus, Poisson's ratio, density etc.are used as an input to estimate the gradation of materials in FGM. 
Manish Bhandari, International Journal of Emerging Trends in Engineering Research, 8(4), April 2020, 969 - 974

\subsection{Gradation}

The laws which are popular in research for gradation of the FGM such as Power law (P-FGM), Sigmoid law (SFGM) and Exponential law (E-FGM) are used in the current work. The material gradation is calculated using the laws and the properties are numerically calculated in the direction of the plate thickness. Some of the volume fraction exponent (n) are selected such as : $\mathrm{n}=0$ (ceramic), 0.1, 0.5, 1, 10, 100 and $n=$ infinity (metal) for P-FGM and S-FGM to conduct performance analysis of FGM plate.

\subsection{Load and dimensions}

A FGM plate (Fig.1) which is simply supported and the ratio of the side dimensions (aspect ratio) is varying in the present simulation. One dimension of the plate is $1 \mathrm{~m}$ and the other dimension is varied. The plate thickness (h) is taken to be $0.02 \mathrm{~m}$. A uniformly distribute load (udl) and a concentrated (point) load (10E6N) is applied for the performance analysis.

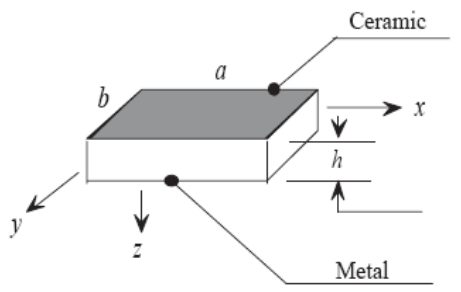

Figure 1: FGM plate

\section{$2.4 \quad$ Numerical method}

Finite element analysis (FEA) is applied to model numerically. Simulation software is used to simulate and compute the parameters such as deflection $\left(\mathrm{u}_{\mathrm{z}}\right)$, tensile stress $\left(\sigma_{\mathrm{x}}\right)$, shear stress $\left(\sigma_{\mathrm{xy}}\right)$, strain $\left(\mathrm{e}_{\mathrm{x}}\right)$ and shear strain $\left(\mathrm{e}_{\mathrm{xy}}\right)$. The parameters are presented in nondimensional terms as follows:

Nondimensional deflection

$\overline{\mathrm{uz}}=\left(\mathrm{u}_{\mathrm{z}} \mathrm{E}_{\mathrm{t}} \mathrm{h}\right) / \mathrm{a}^{2} \mathrm{p}_{\mathrm{o}}$

and nondimensional tensile stress

$\overline{\sigma x}=\sigma_{x} h / p_{o} a$

and nondimensional shear stress

$\overline{\sigma x y}=\sigma_{x y} h / p_{o} a$

\section{RESULTS}

3.1 Effect of udl with variation of Aspect Ratio $(\mathbf{a} / \mathbf{b})$

In this section computational results are presented when the FGM plate is subjected to concentrated or point load of magnitude 1x10E6 Pa. The aspect ratio $(\mathrm{a} / \mathrm{b})$ is varied and non dimensional parameters such as deflection $(\overline{\mathbf{u z}})$, tensile stress $(\overline{\boldsymbol{\sigma} \mathbf{x}})$, shear stress $(\overline{\boldsymbol{\sigma} \mathbf{x y}})$, strain (ex) and shear strain (exy) are calculated. The plate is kept simply supported and the material gradation is done using P-FGM, S-FGM and E-FGM. The volume fraction index ' $\mathrm{n}$ ' is taken as $0.1,0.5,1,10$ and 100 in P-FGM and S-FGM. 'n' $=0$ gives ceramic and 'n' $=\propto$ gives results for metal.

\subsubsection{Non-Dimensional Deflection $(\overline{\mathbf{u z}})$}

Table 1: Non-dimensional deflection $\left(\overline{\mathrm{u}_{\mathrm{z}}}\right)$ under udl for PFGM and E-FGM $(b=1 \mathrm{~m}, \mathrm{~h}=0.02 \mathrm{~m}, \mathrm{udl}=1 \mathrm{x} 10 \mathrm{E} 6 \mathrm{~Pa})$

\begin{tabular}{|c|c|c|c|c|c|c|c|}
\hline & Ceramic & \multicolumn{7}{|c|}{ P-FGM } & Metal \\
\hline $\mathrm{a} / \mathrm{b}$ & $\mathrm{n}=0$ & 0.1 & 0.5 & 1 & 10 & 100 & $\propto$ \\
\hline 0.16 & 0.12 & 0.13 & 0.16 & 0.17 & 0.22 & 0.24 & 0.26 \\
\hline 0.2 & 0.29 & 0.32 & 0.37 & 0.42 & 0.52 & 0.58 & 0.63 \\
\hline 0.25 & 0.7 & 0.77 & 0.9 & 1 & 1.25 & 1.38 & 1.51 \\
\hline 0.5 & 8.83 & 9.72 & 11.34 & 12.61 & 15.78 & 17.45 & 19.04 \\
\hline 0.75 & 29.24 & 32.22 & 37.55 & 41.79 & 52.28 & 57.79 & 63.07 \\
\hline 1 & 56.51 & 62.26 & 72.58 & 80.77 & 101.0 & 111.6 & 121.8 \\
\hline 2 & 139.55 & 153.8 & 179.3 & 199.5 & 249.2 & 275.6 & 301.0 \\
\hline 3 & 167.85 & 185.0 & 215.6 & 239.9 & 299.7 & 331.4 & 362.0 \\
\hline 4 & 175.57 & 193.5 & 225.6 & 251.0 & 313.4 & 346.7 & 378.7 \\
\hline 5 & 177.52 & 195.7 & 228.1 & 253.8 & 316.9 & 350.5 & 382.9 \\
\hline
\end{tabular}

Table 2: Non-dimensional deflection $\left(\overline{\mathrm{u}_{\mathrm{z}}}\right)$ under udl for SFGM $(b=1 \mathrm{~m}, \mathrm{~h}=0.02 \mathrm{~m}, \mathrm{udl}=1 \times 10 \mathrm{E} 6 \mathrm{~Pa})$

\begin{tabular}{|c|c|c|c|c|c|c|}
\hline & \multicolumn{5}{|c|}{ S-FGM } & E-FGM \\
\hline $\mathrm{a} / \mathrm{b}$ & 0.1 & 0.5 & 1 & 10 & 100 & \\
\hline 0.16 & 0.17 & 0.17 & 0.17 & 0.18 & 0.18 & 0.17 \\
\hline 0.2 & 0.4 & 0.41 & 0.42 & 0.44 & 0.44 & 0.42 \\
\hline 0.25 & 0.96 & 0.98 & 1 & 1.06 & 1.06 & 1 \\
\hline 0.5 & 12.12 & 12.34 & 12.61 & 13.34 & 13.38 & 12.56 \\
\hline 0.75 & 40.14 & 40.89 & 41.79 & 44.2 & 44.33 & 41.59 \\
\hline 1 & 77.57 & 79.03 & 80.77 & 85.42 & 85.67 & 80.39 \\
\hline 2 & 191.56 & 195.2 & 199.51 & 211.03 & 211.63 & 198.56 \\
\hline 3 & 230.4 & 234.78 & 239.98 & 253.85 & 252.31 & 238.85 \\
\hline 4 & 241.01 & 245.59 & 251.05 & 265.57 & 266.31 & 249.87 \\
\hline 5 & 243.69 & 248.35 & 253.85 & 268.53 & 269.29 & 252.65 \\
\hline
\end{tabular}

It is evident from Table 1 and 2 that

(a) The non-dimensional deflection increases upto the aspect ratio 3 and it becomes constant beyond the value 3.

(b) The deflection is greatest for pure metal and lowest for pure ceramic. As volume fraction ' $\mathrm{n}$ ' is increased he non-dimensional deflection grows because the stiffness in bending is the greatest for fully ceramic plate, while least for fully metal plate.

\subsubsection{Non-Dimensional Tensile Stress $(\overline{\sigma \mathbf{x}})$}

Table 3: Non-dimensional tensile stress $(\overline{\sigma x})$ under udl for PFGM and E-FGM $(b=1 \mathrm{~m}, \mathrm{~h}=0.02 \mathrm{~m}, \mathrm{udl}=1 \times 10 \mathrm{E} 6 \mathrm{~Pa})$

\begin{tabular}{|c|c|c|c|c|c|c|c|}
\hline & Ceramic & \multicolumn{5}{|c|}{ P-FGM } & Metal \\
\hline $\mathrm{a} / \mathrm{b}$ & $\mathrm{n}=0$ & 0.1 & 0.5 & 1 & 10 & 100 & $\propto$ \\
\hline 0.16 & 0.96 & 1.02 & 1.11 & 1.19 & 1.34 & 1.37 & 1.41 \\
\hline 0.2 & 1.5 & 1.59 & 1.74 & 1.85 & 2.09 & 2.14 & 2.19 \\
\hline 0.25 & 2.32 & 2.47 & 2.7 & 2.87 & 3.25 & 3.33 & 3.4 \\
\hline 0.5 & 7.72 & 8.19 & 8.96 & 9.53 & 10.8 & 11.0 & 11.3 \\
\hline 0.75 & 12.23 & 12.9 & 14.2 & 15.1 & 17.1 & 17.5 & 17.9 \\
\hline 1 & 14.53 & 15.4 & 16.8 & 17.9 & 20.3 & 20.8 & 21.3 \\
\hline 2 & 14.04 & 14.9 & 16.3 & 17.3 & 19.6 & 20.1 & 20.6 \\
\hline 3 & 13.47 & 14.3 & 15.6 & 16.6 & 18.8 & 19.3 & 19.7 \\
\hline 4 & 13.42 & 14.2 & 15.6 & 16.6 & 18.7 & 19.2 & 19.7 \\
\hline 5 & 13.42 & 14.2 & 15.5 & 16.5 & 18.7 & 19.2 & 19.6 \\
\hline
\end{tabular}


Manish Bhandari, International Journal of Emerging Trends in Engineering Research, 8(4), April 2020, 969 - 974

Table 4: Non-dimensional tensile stress $(\overline{\sigma x})$ under udl for $S$ FGM $(b=1 \mathrm{~m}, \mathrm{~h}=0.02 \mathrm{~m}, \mathrm{udl}=1 \mathrm{x} 10 \mathrm{E} 6 \mathrm{~Pa})$

\begin{tabular}{|c|c|c|c|c|c|c|}
\hline & \multicolumn{5}{|c|}{ S-FGM } & E-FGM \\
\hline $\mathrm{a} / \mathrm{b}$ & 0.1 & 0.5 & 1 & 10 & 100 & \\
\hline 0.16 & 1.13 & 1.17 & 1.19 & 1.24 & 1.26 & 1.21 \\
\hline 0.2 & 1.76 & 1.82 & 1.85 & 1.94 & 1.97 & 1.88 \\
\hline 0.25 & 2.73 & 2.82 & 2.87 & 3.01 & 3.05 & 2.92 \\
\hline 0.5 & 9.07 & 9.38 & 9.53 & 9.99 & 10.15 & 9.71 \\
\hline 0.75 & 14.38 & 14.87 & 15.1 & 15.85 & 16.1 & 15.4 \\
\hline 1 & 17.09 & 17.68 & 17.9 & 18.86 & 19.17 & 18.32 \\
\hline 2 & 16.52 & 17.09 & 17.3 & 18.25 & 18.55 & 17.71 \\
\hline 3 & 15.84 & 16.39 & 16.6 & 17.49 & 17.77 & 16.98 \\
\hline 4 & 15.79 & 16.33 & 16.6 & 17.42 & 17.71 & 16.92 \\
\hline 5 & 15.78 & 16.32 & 16.5 & 17.42 & 17.7 & 16.91 \\
\hline
\end{tabular}

When we closely observe the Table 3 and 4 it is revealed that

(a) The non-dimensional tensile stress increases upto aspect ratio 2 and beyond 2 it starts reducing. The nondimensional tensile stress increases steeply between aspect ratio 0.25 to 2 . The non-dimensional tensile stress reduces as the aspect ratio increases and it becomes constant as the aspect ratio is increased beyond the value 4 .

(b) The non-dimensional tensile stress is highest for pure metal $(n=\infty)$ and lowest for pure ceramic $(n=0)$.

\subsubsection{Non-dimensional Shear Stress $(\overline{\sigma x} \mathbf{y})$}

Table 5: Non-dimensional shear stress ( $\overline{\sigma x y})$ under udl for PFGM and E-FGM $(b=1 \mathrm{~m}, \mathrm{~h}=0.02 \mathrm{~m}, \mathrm{udl}=1 \times 10 \mathrm{E} 6 \mathrm{~Pa})$

\begin{tabular}{|c|c|c|c|c|c|c|c|}
\hline & Ceramic & \multicolumn{7}{|c|}{ P-FGM } & Metal \\
\hline $\mathrm{a} / \mathrm{b}$ & $\mathrm{n}=0$ & 0.1 & 0.5 & 1 & 10 & 100 & $\propto$ \\
\hline 0.16 & 0.3 & 0.32 & 0.35 & 0.37 & 0.41 & 0.42 & 0.42 \\
\hline 0.2 & 0.49 & 0.52 & 0.57 & 0.6 & 0.68 & 0.68 & 0.69 \\
\hline 0.25 & 0.79 & 0.84 & 0.92 & 0.97 & 1.1 & 1.11 & 1.13 \\
\hline 0.5 & 3.34 & 3.54 & 3.87 & 4.1 & 4.65 & 4.74 & 4.83 \\
\hline 0.75 & 6.72 & 7.14 & 7.79 & 8.27 & 9.37 & 9.55 & 9.73 \\
\hline 1 & 9.62 & 10.2 & 11.1 & 11.8 & 13.4 & 13.6 & 13.9 \\
\hline 2 & 13.83 & 14.6 & 16.0 & 17.0 & 19.3 & 19.7 & 20.1 \\
\hline 3 & 13.97 & 14.8 & 16.2 & 17.2 & 19.5 & 19.9 & 20.4 \\
\hline 4 & 13.73 & 14.5 & 15.9 & 16.9 & 19.2 & 19.6 & 20.0 \\
\hline 5 & 13.54 & 14.3 & 15.7 & 16.7 & 18.8 & 19.2 & 19.6 \\
\hline
\end{tabular}

Table 6: Non-dimensional shear stress ( $\overline{\sigma x y})$ under udl for SFGM $(b=1 \mathrm{~m}, \mathrm{~h}=0.02 \mathrm{~m}, \mathrm{udl}=1 \times 10 \mathrm{E} 6 \mathrm{~Pa})$

\begin{tabular}{|c|c|c|c|c|c|c|}
\hline & \multicolumn{7}{|c|}{ S-FGM } & E-FGM \\
\hline $\mathrm{a} / \mathrm{b}$ & 0.1 & 0.5 & 1 & 10 & 100 & \\
\hline 0.16 & 0.35 & 0.36 & 0.37 & 0.37 & 0.37 & 0.37 \\
\hline 0.2 & 0.57 & 0.59 & 0.6 & 0.61 & 0.61 & 0.61 \\
\hline 0.25 & 0.93 & 0.96 & 0.97 & 0.99 & 0.99 & 0.99 \\
\hline 0.5 & 3.92 & 4.04 & 4.1 & 4.16 & 4.18 & 4.18 \\
\hline 0.75 & 7.89 & 8.15 & 8.27 & 8.39 & 8.44 & 8.42 \\
\hline 1 & 11.3 & 11.67 & 11.84 & 12.03 & 12.09 & 12.06 \\
\hline 2 & 16.24 & 16.78 & 17.04 & 17.33 & 17.43 & 17.36 \\
\hline 3 & 16.41 & 16.95 & 17.21 & 17.5 & 17.6 & 17.54 \\
\hline 4 & 16.13 & 16.66 & 16.92 & 17.2 & 17.3 & 17.24 \\
\hline 5 & 15.91 & 16.44 & 16.71 & 17.02 & 17.12 & 17.01 \\
\hline
\end{tabular}

By looking Table 5 and Table 6 it is clear that

a.) the non-dimensional shear stress ( $\sigma x y)$ increases as the aspect ratio is increased, it reaches maximum value at aspect ratio 3, it reduces as the aspect ratio increases beyond 3 .

b) The non-dimensional shear stress ( $\sigma x y)$ has a steep decline between aspect ratio 3 to 4 and a gradual decline after aspect ratio 4 .

c) The shear stress ( $\sigma x y)$ is most for pure metal $(n=\infty)$ plate and minimum for the case of pure ceramic plate (n $=0)$.

\subsubsection{Strain $\left(\mathbf{e}_{\mathrm{x}}\right)$}

Table 7: Strain $\left(\mathrm{e}_{\mathrm{x}} \mathrm{x} 1000\right)$ under udl for P-FGM and E-FGM $(b=1 \mathrm{~m}, \mathrm{~h}=0.02 \mathrm{~m}, \mathrm{udl}=1 \mathrm{x} 10 \mathrm{E} 6 \mathrm{~Pa})$

\begin{tabular}{|c|c|c|c|c|c|c|c|}
\hline & Ceramic & \multicolumn{5}{|c|}{ P-FGM } & Metal \\
\hline $\mathrm{a} / \mathrm{b}$ & $\mathrm{n}=0$ & 0.1 & 0.5 & 1 & 10 & 100 & $\propto$ \\
\hline 0.16 & 0.29 & 0.31 & 0.34 & 0.36 & 0.47 & 0.55 & 0.62 \\
\hline 0.2 & 0.45 & 0.48 & 0.53 & 0.57 & 0.74 & 0.86 & 0.97 \\
\hline 0.25 & 0.7 & 0.74 & 0.82 & 0.88 & 1.14 & 1.33 & 1.51 \\
\hline 0.5 & 2.21 & 2.35 & 2.58 & 2.77 & 3.62 & 4.22 & 4.76 \\
\hline 0.75 & 3.19 & 3.4 & 3.74 & 4.01 & 5.25 & 6.11 & 6.89 \\
\hline 1 & 3.37 & 3.58 & 3.95 & 4.24 & 5.54 & 6.44 & 7.27 \\
\hline 2 & 2.28 & 2.43 & 2.67 & 2.87 & 3.75 & 4.36 & 4.92 \\
\hline 3 & 2.15 & 2.29 & 2.52 & 2.71 & 3.54 & 4.11 & 4.64 \\
\hline 4 & 2.14 & 2.27 & 2.51 & 2.69 & 3.52 & 4.09 & 4.61 \\
\hline 5 & 2.13 & 2.27 & 2.5 & 2.68 & 3.5 & 4.07 & 4.59 \\
\hline
\end{tabular}

Table 8: Strain $\left(e_{x} x 1000\right)$ under udl for S-FGM $(b=1 m$, $\mathrm{h}=0.02 \mathrm{~m}, \mathrm{udl}=1 \times 10 \mathrm{E} 6 \mathrm{~Pa})$

\begin{tabular}{|c|c|c|c|c|c|c|}
\hline & \multicolumn{5}{|c|}{ S-FGM } & E-FGM \\
\hline $\mathrm{a} / \mathrm{b}$ & 0.1 & 0.5 & 1 & 10 & 100 & \\
\hline 0.16 & 0.35 & 0.36 & 0.36 & 0.38 & 0.38 & 0.37 \\
\hline 0.2 & 0.55 & 0.56 & 0.57 & 0.59 & 0.59 & 0.58 \\
\hline 0.25 & 0.85 & 0.87 & 0.88 & 0.91 & 0.92 & 0.9 \\
\hline 0.5 & 2.67 & 2.74 & 2.77 & 2.88 & 2.91 & 2.84 \\
\hline 0.75 & 3.87 & 3.97 & 4.01 & 4.17 & 4.22 & 4.12 \\
\hline 1 & 4.1 & 4.19 & 4.24 & 4.4 & 4.45 & 4.35 \\
\hline 2 & 2.78 & 2.84 & 2.87 & 2.98 & 3.01 & 2.95 \\
\hline 3 & 2.62 & 2.68 & 2.71 & 2.81 & 2.84 & 2.78 \\
\hline 4 & 2.6 & 2.66 & 2.69 & 2.79 & 2.82 & 2.76 \\
\hline 5 & 2.59 & 2.65 & 2.68 & 2.78 & 2.81 & 2.75 \\
\hline
\end{tabular}

The facts can be disclosed by looking the Tables 7 and 8 that

(a) The strain (ex) grows as the aspect ratio grows, it attains most value at aspect ratio 1 , then it reduces as the aspect ratio crosses 1 and it becomes constant as aspect ratio is gone beyond 3 . This suggests that maximum strain is obtained in case of square plate.

(b) The strain (ex) is maximum for pure metal $(n=\infty)$ and minimum for pure ceramic $(n=0)$ plate.

\subsubsection{Shear Strain $\left(\mathbf{e}_{\mathrm{xy}}\right)$}

The facts can be disclosed by looking the Tables 9 and 10 that

(a) The shear strain (exy) increases as the aspect ratio is increased, it reaches maximum value at aspect ratio 3 , it becomes constant as the aspect ratio is increased beyond the value 4 .

(b) The shear strain (exy) is maximum for metal $(n=\infty)$ plate and minimum for pure ceramic $(n=0)$ plate

Table 9: Shear Strain $\left(\mathrm{e}_{\mathrm{xy}} \mathrm{x} 1000\right)$ under udl for P-FGM and EFGM $(b=1 \mathrm{~m}, \mathrm{~h}=0.02 \mathrm{~m}, \mathrm{udl}=1 \times 10 \mathrm{E} 6 \mathrm{~Pa})$

\begin{tabular}{|c|c|c|c|c|c|c|c|}
\hline & Ceramic & \multicolumn{7}{|c|}{ P-FGM } & Metal \\
\hline $\mathrm{a} / \mathrm{b}$ & $\mathrm{n}=0$ & 0.1 & 0.5 & 1 & 10 & 100 & $\propto$ \\
\hline 0.16 & 0.26 & 0.3 & 0.37 & 0.42 & 0.49 & 0.52 & 0.56 \\
\hline 0.2 & 0.42 & 0.48 & 0.6 & 0.69 & 0.81 & 0.86 & 0.91 \\
\hline 0.25 & 0.68 & 0.78 & 0.97 & 1.11 & 1.3 & 1.38 & 1.47 \\
\hline 0.5 & 2.87 & 3.29 & 4.04 & 4.64 & 5.55 & 5.86 & 6.2 \\
\hline 0.75 & 5.79 & 6.62 & 8.14 & 9.33 & 11.16 & 11.8 & 12.49 \\
\hline 1 & 8.29 & 9.47 & 11.6 & 13.3 & 15.96 & 16.88 & 17.87 \\
\hline 2 & 11.91 & 13.6 & 16.7 & 19.1 & 22.97 & 24.26 & 25.68 \\
\hline 3 & 12.03 & 13.4 & 16.8 & 19.3 & 23.25 & 24.55 & 25.94 \\
\hline 4 & 11.82 & 13.5 & 16.5 & 19 & 22.86 & 24.13 & 25.5 \\
\hline 5 & 11.66 & 13.3 & 16.3 & 18.7 & 22.45 & 23.74 & 25.15 \\
\hline
\end{tabular}


Manish Bhandari, International Journal of Emerging Trends in Engineering Research, 8(4), April 2020, 969 - 974

Table 10: Shaer Strain $\left(\mathrm{e}_{\mathrm{xy}} \mathrm{x}\right.$ 1000) under udl for S-FGM $(\mathrm{b}=1 \mathrm{~m}, \mathrm{~h}=0.02 \mathrm{~m}, \mathrm{udl}=1 \times 10 \mathrm{E} 6 \mathrm{~Pa})$

\begin{tabular}{|c|c|c|c|c|c|c|}
\hline & \multicolumn{7}{|c|}{ S-FGM } & $\begin{array}{c}\text { E- } \\
\text { FGM }\end{array}$ \\
\hline $\mathrm{a} / \mathrm{b}$ & 0.1 & 0.5 & 1 & 10 & 100 & \\
\hline 0.16 & 0.37 & 0.4 & 0.42 & 0.47 & 0.47 & 0.43 \\
\hline 0.2 & 0.6 & 0.65 & 0.69 & 0.77 & 0.77 & 0.71 \\
\hline 0.25 & 0.98 & 1.05 & 1.11 & 1.24 & 1.24 & 1.14 \\
\hline 0.5 & 4.11 & 4.39 & 4.64 & 5.17 & 5.19 & 4.8 \\
\hline 0.75 & 8.27 & 8.84 & 9.33 & 10.4 & 10.45 & 9.67 \\
\hline 1 & 11.83 & 12.65 & 13.35 & 14.88 & 14.95 & 13.83 \\
\hline 2 & 16.99 & 18.15 & 19.15 & 21.32 & 21.42 & 19.83 \\
\hline 3 & 17.16 & 18.33 & 19.34 & 21.53 & 21.25 & 20.03 \\
\hline 4 & 16.87 & 18.01 & 19 & 21.15 & 21.25 & 19.68 \\
\hline 5 & 16.64 & 17.78 & 18.77 & 20.9 & 21 & 19.43 \\
\hline
\end{tabular}

It is exhibited that the P-FGM and E-FGM characteristics are closer to each other when we compare to that of S-FGM. The S-FGM characteristics are smoother as compared to P-FGM and E-FGM. Also the non-dimensional tensile stress, shear stress, nondimensional deflection, transverse strain and shear strain for the three FGM's are in between that of ceramic and metal.

\subsection{Effect of point load with variation of Aspect Ratio (a/b)}

In this section computational results are presented when the FGM plate is subjected to concentrated or point load of magnitude $1 \times 10 \mathrm{E} 6 \mathrm{~Pa}$. The aspect ratio $(\mathrm{a} / \mathrm{b})$ is varied and non dimensional parameters such as deflection $(\overline{\mathbf{u z}})$, tensile stress $(\overline{\boldsymbol{\sigma} \mathbf{x}})$, shear stress $(\overline{\boldsymbol{\sigma} \mathbf{x y}})$, strain $\left(\mathrm{e}_{\mathrm{x}}\right)$ and shear strain $\left(\mathrm{e}_{\mathrm{xy}}\right)$ are calculated. The plate is kept simply supported and the material gradation is done using P-FGM, S-FGM and E-FGM. The volume fraction index ' $n$ ' is taken as $0.1,0.5,1,10$ and 100 in P-FGM and S-FGM. ' $n$ ' $=0$ gives ceramic and ' $n$ ' $=\propto$ gives results for metal.

\subsubsection{Non-Dimensional Deflection $(\overline{\mathbf{u z}})$}

Table 11: Non-dimensional deflection $\left(\overline{\mathrm{u}_{\mathrm{z}}}\right)$ under point load (10E6 Pa) for P-FGM and E-FGM ( $b=1 \mathrm{~m}, \mathrm{~h}=0.02 \mathrm{~m}$ )

\begin{tabular}{|c|c|c|c|c|c|c|c|}
\hline & Ceramic & \multicolumn{5}{|c|}{ P-FGM } & Metal \\
\hline $\mathrm{a} / \mathrm{b}$ & $\mathrm{n}=0$ & 0.1 & 0.5 & 1 & 10 & 100 & $\propto$ \\
\hline 0.16 & 1.16 & 1.27 & 1.48 & 1.53 & 2.08 & 2.29 & 2.49 \\
\hline 0.2 & 2.13 & 2.34 & 2.73 & 2.82 & 3.82 & 4.21 & 4.59 \\
\hline 0.25 & 3.99 & 4.39 & 5.12 & 5.28 & 7.14 & 7.89 & 8.6 \\
\hline 0.5 & 29.25 & 32.23 & 37.56 & 38.71 & 52.3 & 57.81 & 63.09 \\
\hline 0.75 & 85.72 & 94.45 & 110.1 & 113.4 & 153.2 & 169.4 & 184.9 \\
\hline 1 & 161.9 & 178.41 & 207.97 & 214.2 & 289.4 & 319.9 & 349.2 \\
\hline 2 & 456.8 & 503.46 & 586.9 & 604.7 & 816.0 & 902.3 & 985.3 \\
\hline 3 & 700.8 & 772.32 & 900.3 & 927.61 & 1251. & 1383.8 & 1511.3 \\
\hline 4 & 935.0 & 1030.7 & 1201. & 1238. & 1669 & 1846 & 2017 \\
\hline 5 & 1168.6 & 1288.2 & 1501 & 1547 & 2086 & 2307 & 2520 \\
\hline
\end{tabular}

Table 12: Non-dimensional deflection $\left(\overline{\mathrm{u}_{\mathrm{z}}}\right)$ under point load $(10 \mathrm{E} 6 \mathrm{~Pa})$ for $\mathrm{S}-\mathrm{FGM}(\mathrm{b}=1 \mathrm{~m}, \mathrm{~h}=0.02 \mathrm{~m})$

\begin{tabular}{|c|c|c|c|c|c|c|}
\hline & \multicolumn{7}{|c|}{ S-FGM } & E-FGM \\
\hline $\mathrm{a} / \mathrm{b}$ & 0.1 & 0.5 & 1 & 10 & 100 & \\
\hline 0.16 & 1.59 & 1.62 & 1.65 & 1.74 & 1.75 & 1.64 \\
\hline 0.2 & 2.92 & 2.98 & 3.04 & 3.22 & 3.23 & 3.03 \\
\hline 0.25 & 5.47 & 5.58 & 5.7 & 6.03 & 6.04 & 5.67 \\
\hline 0.5 & 40.15 & 40.9 & 41.8 & 44.21 & 44.34 & 41.61 \\
\hline 0.75 & 117.67 & 119.88 & 122.52 & 129.57 & 129.95 & 121.94 \\
\hline 1 & 222.25 & 226.44 & 231.43 & 244.75 & 245.47 & 230.34 \\
\hline 2 & 627.04 & 638.97 & 653.08 & 690.79 & 692.77 & 649.98 \\
\hline 3 & 961.8 & 980.11 & 1001.82 & 1059.7 & 1053.27 & 997.09 \\
\hline 4 & 1283.62 & 1308.02 & 1337.09 & 1414.4 & 1418.34 & 1330.77 \\
\hline 5 & 1604.18 & 1634.83 & 1671.06 & 1767.71 & 1772.69 & 1663.15 \\
\hline
\end{tabular}

It is evident from Table 11 and 12 that

(a) The non-dimensional deflection increases upto the aspect ratio 3 and it becomes constant as the aspect ratio is increased beyond the value 3 .

(b) The deflection is greatest for pure metal and lowest for pure ceramic. As volume fraction ' $n$ ' is increased he non-dimensional deflection grows because the stiffness in bending is the greatest for fully ceramic plate, while least for fully metal plate.

\subsubsection{Non-Dimensional Tensile Stress $(\overline{\boldsymbol{\sigma} \mathbf{x}})$}

Table 13: Non-dimensional Tensile Stress $(\overline{\sigma x})$ under point load $(10 \mathrm{E} 6 \mathrm{~Pa})$ for P-FGM and E-FGM $(\mathrm{b}=1 \mathrm{~m}, \mathrm{~h}=0.02 \mathrm{~m})$

\begin{tabular}{|c|c|c|c|c|c|c|c|}
\hline & & \multicolumn{7}{|c|}{ P-FGM } & Metal \\
\hline $\mathrm{a} / \mathrm{b}$ & $\mathrm{n}=0$ & 0.1 & 0.5 & 1 & 10 & 100 & $\propto$ \\
\hline 0.16 & 19.37 & 20.57 & 22.5 & 23.93 & 27.09 & 27.73 & 28.37 \\
\hline 0.2 & 25.21 & 26.77 & 29.27 & 31.12 & 35.25 & 36.07 & 36.91 \\
\hline 0.25 & 32.68 & 34.7 & 37.94 & 40.34 & 45.7 & 46.76 & 47.86 \\
\hline 0.5 & 71.08 & 75.47 & 82.53 & 87.76 & 99.45 & 101.2 & 104.24 \\
\hline 0.75 & 107.1 & 113.6 & 124.4 & 132.6 & 149.3 & 153.6 & 157.28 \\
\hline 1 & 138.4 & 147.9 & 160.5 & 171.4 & 193.4 & 198.8 & 203.45 \\
\hline 2 & 240.3 & 255.2 & 279.7 & 297.6 & 336.3 & 344.6 & 352.99 \\
\hline 3 & 329.1 & 349.4 & 382.6 & 407.2 & 460.6 & 471.5 & 483.17 \\
\hline 4 & 409.0 & 434.4 & 475.5 & 505.6 & 572.2 & 586.1 & 600.24 \\
\hline 5 & 481.4 & 511.4 & 559.5 & 595.2 & 673.7 & 689.2 & 706.53 \\
\hline
\end{tabular}

Table 14: Non-dimensional Tensile Stress $(\overline{\sigma x})$ under point load (10E6 Pa) for S-FGM ( $b=1 \mathrm{~m}, \mathrm{~h}=0.02 \mathrm{~m})$

\begin{tabular}{|c|c|c|c|c|c|c|}
\hline & \multicolumn{5}{|c|}{ S-FGM } & E-FGM \\
\hline $\mathrm{a} / \mathrm{b}$ & 0.1 & 0.5 & 1 & 10 & 100 & \\
\hline 0.16 & 22.78 & 23.55 & 23.93 & 25.09 & 25.5 & 24.39 \\
\hline 0.2 & 29.63 & 30.63 & 31.12 & 32.64 & 33.16 & 31.72 \\
\hline 0.25 & 38.41 & 39.71 & 40.34 & 42.29 & 42.96 & 41.12 \\
\hline 0.5 & 83.57 & 86.39 & 87.76 & 92.01 & 93.47 & 89.47 \\
\hline 0.75 & 125.8 & 130.7 & 132.6 & 138.8 & 141.1 & 134.9 \\
\hline 1 & 162.9 & 168.9 & 171.4 & 179.7 & 182.1 & 174.5 \\
\hline 2 & 282.2 & 292.1 & 297.6 & 312.9 & 317.5 & 303.1 \\
\hline 3 & 387.5 & 400.6 & 407.2 & 427.5 & 434.3 & 414.9 \\
\hline 4 & 481.0 & 497.4 & 505.6 & 530.3 & 539.5 & 515.4 \\
\hline 5 & 566.2 & 585.8 & 595.2 & 624.9 & 634.7 & 606.7 \\
\hline
\end{tabular}

When we closely observe the Table 13 and 14 it is revealed that

(a) Since the effect of concentrated load is somewhat different as compared to udl, we find that the nondimensional tensile stress increases as the aspect ratio increases.

(b) The non-dimensional tensile stress is highest for pure metal $(n=\infty)$ and lowest for pure ceramic $(n=0)$.

\subsubsection{Non-Dimensional Shear Stress $(\overline{\sigma x} y)$}

Table 15: Non-dimensional shear Stress $(\overline{\sigma x y})$ under point load $(10 \mathrm{E} 6 \mathrm{~Pa})$ for P-FGM and E-FGM $(\mathrm{b}=1 \mathrm{~m}, \mathrm{~h}=0.02 \mathrm{~m})$

\begin{tabular}{|c|c|c|c|c|c|c|c|}
\hline & Ceramic & \multicolumn{5}{|c|}{ P-FGM } & Metal \\
\hline $\mathrm{a} / \mathrm{b}$ & $\mathrm{n}=0$ & 0.1 & 0.5 & 1 & 10 & 100 & $\propto$ \\
\hline 0.16 & 1.72 & 1.83 & 1.99 & 2.1 & 2.37 & 2.39 & 2.41 \\
\hline 0.2 & 2.18 & 2.32 & 2.53 & 2.67 & 3.01 & 3.04 & 3.07 \\
\hline 0.25 & 2.75 & 2.92 & 3.19 & 3.37 & 3.8 & 3.86 & 3.91 \\
\hline 0.5 & 6.01 & 6.38 & 6.96 & 7.39 & 8.38 & 8.54 & 8.7 \\
\hline 0.75 & 12.25 & 13 & 14.9 & 15.6 & 17.8 & 17.3 & 17.7 \\
\hline 1 & 18.42 & 19.5 & 21.6 & 22.7 & 25.8 & 26.6 & 26.6 \\
\hline 2 & 24.12 & 25.2 & 27.9 & 29.3 & 33.6 & 34.9 & 35.1 \\
\hline 3 & 33.57 & 35.4 & 38.4 & 41.7 & 46.2 & 47.9 & 49.0 \\
\hline 4 & 44.45 & 47.8 & 51.5 & 54.6 & 62.5 & 63.6 & 65 \\
\hline 5 & 55.43 & 58.2 & 64.4 & 68.9 & 77.3 & 78.9 & 80.5 \\
\hline
\end{tabular}


Manish Bhandari, International Journal of Emerging Trends in Engineering Research, 8(4), April 2020, 969 - 974

Table 16: Non-dimensional shear Stress $(\overline{\sigma x y})$ under point load $(10 \mathrm{E} 6 \mathrm{~Pa})$ for $\mathrm{S}-\mathrm{FGM}(\mathrm{b}=1 \mathrm{~m}, \mathrm{~h}=0.02 \mathrm{~m})$

\begin{tabular}{|c|c|c|c|c|c|c|}
\hline & \multicolumn{5}{|c|}{ S-FGM } & E-FGM \\
\hline $\mathrm{a} / \mathrm{b}$ & 0.1 & 0.5 & 1 & 10 & 100 & \\
\hline 0.16 & 2.01 & 2.07 & 2.1 & 2.14 & 2.15 & 2.14 \\
\hline 0.2 & 2.55 & 2.63 & 2.67 & 2.71 & 2.72 & 2.71 \\
\hline 0.25 & 3.22 & 3.32 & 3.37 & 3.43 & 3.45 & 3.43 \\
\hline 0.5 & 7.06 & 7.28 & 7.39 & 7.5 & 7.53 & 7.52 \\
\hline 0.75 & 14.38 & 14.84 & 15.06 & 15.29 & 15.37 & 15.34 \\
\hline 1 & 21.63 & 22.33 & 22.67 & 23.03 & 23.15 & 23.08 \\
\hline 2 & 28.34 & 29.27 & 29.73 & 30.23 & 30.4 & 30.28 \\
\hline 3 & 39.44 & 40.74 & 41.37 & 42.06 & 42.29 & 42.15 \\
\hline 4 & 52.22 & 53.93 & 54.76 & 55.69 & 56 & 55.81 \\
\hline 5 & 65.13 & 67.31 & 68.39 & 69.66 & 70.08 & 69.63 \\
\hline
\end{tabular}

By looking Table 15 and Table 16 it is clear that (a) Since the effect of concentrated load is somewhat different as compared to udl, we find that the nondimensional shear stress increases as the aspect ratio increases.

(b) The non-dimensional tensile stress is highest for pure metal $(n=\infty)$ and lowest for pure ceramic $(n=0)$.

\subsubsection{Strain $\left(e_{x}\right)$}

Table 17: Strain $\left(\mathrm{e}_{\mathrm{x}} \mathrm{x} 1000\right)$ under point load (10E6 Pa) for PFGM and E-FGM $(b=1 \mathrm{~m}, \mathrm{~h}=0.02 \mathrm{~m})$

\begin{tabular}{|c|c|c|c|c|c|c|c|}
\hline & Ceramic & \multicolumn{7}{|c|}{ P-FGM } & Metal \\
\hline $\mathrm{a} / \mathrm{b}$ & $\mathrm{n}=0$ & 0.1 & 0.5 & 1 & 10 & 100 & $\propto$ \\
\hline 0.16 & 4.99 & 5.31 & 5.85 & 6.27 & 8.19 & 9.54 & 10.77 \\
\hline 0.2 & 6.45 & 6.86 & 7.55 & 8.1 & 10.58 & 12.31 & 13.91 \\
\hline 0.25 & 8.3 & 8.82 & 9.71 & 10.41 & 13.61 & 15.85 & 17.9 \\
\hline 0.5 & 17.58 & 18.69 & 20.57 & 22.06 & 28.85 & 33.59 & 37.92 \\
\hline 0.75 & 25.69 & 27.32 & 30.09 & 32.27 & 42.19 & 49.1 & 55.42 \\
\hline 1 & 32.1 & 34.15 & 37.63 & 40.37 & 52.76 & 61.36 & 69.24 \\
\hline 2 & 51.51 & 54.81 & 60.41 & 64.83 & 84.75 & 98.51 & 111.12 \\
\hline 3 & 68.83 & 73.22 & 80.67 & 86.56 & 113.1 & 131.6 & 148.47 \\
\hline 4 & 84.25 & 89.62 & 98.73 & 105.92 & 138.5 & 161.07 & 181.73 \\
\hline 5 & 97.94 & 104.19 & 114.78 & 123.14 & 161 & 187.23 & 211.28 \\
\hline
\end{tabular}

Table 18: Strain $\left(e_{x} x 1000\right)$ under point load (10E6 Pa) for $S$ FGM $(b=1 \mathrm{~m}, \mathrm{~h}=0.02 \mathrm{~m})$

\begin{tabular}{|c|c|c|c|c|c|c|}
\hline & \multicolumn{5}{|c|}{ S-FGM } & E-FGM \\
\hline $\mathrm{a} / \mathrm{b}$ & 0.1 & 0.5 & 1 & 10 & 100 & \\
\hline 0.16 & 6.06 & 6.2 & 6.27 & 6.51 & 6.59 & 6.44 \\
\hline 0.2 & 7.82 & 8 & 8.1 & 8.41 & 8.51 & 8.31 \\
\hline 0.25 & 10.05 & 10.29 & 10.41 & 10.82 & 10.94 & 10.69 \\
\hline 0.5 & 21.27 & 21.79 & 22.06 & 22.92 & 23.19 & 22.64 \\
\hline 0.75 & 31.15 & 31.89 & 32.27 & 33.51 & 33.9 & 33.12 \\
\hline 1 & 39.06 & 39.93 & 40.37 & 41.89 & 42.37 & 41.44 \\
\hline 2 & 62.8 & 64.15 & 64.83 & 67.25 & 68.01 & 66.56 \\
\hline 3 & 83.74 & 85.61 & 86.56 & 89.83 & 90.85 & 88.86 \\
\hline 4 & 102.43 & 104.74 & 105.92 & 109.94 & 111.2 & 108.74 \\
\hline 5 & 119.09 & 121.77 & 123.14 & 127.81 & 129.28 & 126.41 \\
\hline
\end{tabular}

The facts can be disclosed by looking the Tables 17 and 18 that

(a) The strain (ex) grows as the aspect ratio grows, since the effect of point load is differing than the effect of udl.

(b) The strain (ex) is maximum for pure metal $(\mathrm{n}=\infty)$ and minimum for pure ceramic $(\mathrm{n}=0)$ plate.

(c) The strain (ex) for P-FGM is fairly distributed for different values of volume fraction exponent ' $n$ ' whereas in case of S-FGM the volume fraction exponent ' $n$ ' has little influence on it.

\subsubsection{Shear Strain $\left(\mathbf{e}_{\mathrm{xy}}\right)$}

The facts can be disclosed by looking the Tables 19 and 20 that

(a) The shear strain (exy) increases as the aspect ratio is increased and reason is attributed with the difference in effect of point load and udl.

(b) The shear strain (exy) is maximum for metal $(\mathrm{n}=\infty)$ plate and minimum for pure ceramic $(n=0)$ plate.

Table 19: Shear Strain $\left(\mathrm{e}_{\mathrm{xy}} \mathrm{x} 1000\right)$ under point load (10E6 Pa) for P-FGM and E-FGM $(b=1 \mathrm{~m}, \mathrm{~h}=0.02 \mathrm{~m})$

\begin{tabular}{|c|l|l|l|l|l|l|l|}
\hline & Ceramic & \multicolumn{5}{|c|}{ P-FGM } & Metal \\
\hline $\mathrm{a} / \mathrm{b}$ & $\mathrm{n}=0$ & 0.1 & 0.5 & 1 & 10 & 100 & $\propto$ \\
\hline 0.16 & 1.47 & 1.69 & 2.09 & 2.4 & 2.81 & 2.99 & 3.18 \\
\hline 0.2 & 1.87 & 2.15 & 2.64 & 3.03 & 3.57 & 3.79 & 4.03 \\
\hline 0.25 & 2.36 & 2.71 & 3.34 & 3.82 & 4.51 & 4.78 & 5.08 \\
\hline 0.5 & 5.16 & 5.9 & 7.25 & 8.32 & 9.95 & 10.52 & 11.12 \\
\hline 0.75 & 10.54 & 12.5 & 14.1 & 16.9 & 20.2 & 21.48 & 22.73 \\
\hline 1 & 15.85 & 18.3 & 22.7 & 25.5 & 30.4 & 32.3 & 34.2 \\
\hline 2 & 20.74 & 23.7 & 29.1 & 33.3 & 40.2 & 42.27 & 44.74 \\
\hline 3 & 28.9 & 33.1 & 40.5 & 46.4 & 55.7 & 58.98 & 62.34 \\
\hline 4 & 38.28 & 43.7 & 53.4 & 61.5 & 74.2 & 78.14 & 82.57 \\
\hline 5 & 47.73 & 54.5 & 67.0 & 76.3 & 91.9 & 97.2 & 102.96 \\
\hline
\end{tabular}

Table 20: Shear Strain $\left(\mathrm{e}_{\mathrm{xy}} \mathrm{x} 1000\right)$ under point load (10E6 Pa) for S-FGM $(b=1 \mathrm{~m}, \mathrm{~h}=0.02 \mathrm{~m})$

\begin{tabular}{|c|l|l|l|l|l|l|}
\hline & \multicolumn{5}{|c|}{ S-FGM } & E-FGM \\
\hline $\mathrm{a} / \mathrm{b}$ & 0.1 & 0.5 & \multicolumn{1}{|c|}{1} & \multicolumn{1}{c|}{10} & 100 & \\
\hline 0.16 & 2.11 & 2.26 & 2.4 & 2.68 & 2.69 & 2.48 \\
\hline 0.2 & 2.67 & 2.87 & 3.03 & 3.39 & 3.4 & 3.13 \\
\hline 0.25 & 3.37 & 3.61 & 3.82 & 4.27 & 4.29 & 3.95 \\
\hline 0.5 & 7.37 & 7.88 & 8.32 & 9.27 & 9.32 & 8.62 \\
\hline 0.75 & 15.05 & 16.1 & 16.99 & 18.93 & 19.02 & 17.6 \\
\hline 1 & 22.64 & 24.2 & 25.55 & 28.47 & 28.6 & 26.46 \\
\hline 2 & 29.6 & 31.63 & 33.36 & 37.15 & 37.32 & 34.56 \\
\hline 3 & 41.24 & 44.05 & 46.47 & 51.73 & 51.06 & 48.14 \\
\hline 4 & 54.62 & 58.33 & 61.52 & 68.49 & 68.81 & 63.73 \\
\hline 5 & 68.12 & 72.81 & 76.83 & 85.57 & 85.96 & 79.54 \\
\hline
\end{tabular}

It is exhibited that the P-FGM and E-FGM characteristics are closer to each other when we compare to that of S-FGM. The S-FGM characteristics are smoother as compared to P-FGM and E-FGM. Also the non-dimensional tensile stress, shear stress, nondimensional deflection, transverse strain and shear strain for the three FGM's are in between that of ceramic and metal.

\section{CONCLUSIONS AND FUTURE SCOPE}

A FGM plate is subjected to transverse static udl and concentrated loading. The aspect ratio is varied and non dimensional parameters are computed using simulation software. It has been observed that the response in bending of the FG plate is in between to those of the pure metal and pure ceramic plate. The non-dimensional deflection is largest for pure metal $(n=\infty)$ and lowest for pure ceramic $(n=0)$ plate. As the volume fraction ' $n$ ' increases the non-dimensional deflection grows. The non-dimensional tensile stress diminishes as the aspect ratio grows and it shows constant behaviour as the aspect ratio increases further beyond. 6. The nondimensional shear stress $\left(\sigma_{\mathrm{xy}}\right)$ increases with the aspect ratio, it reaches highest value at aspect ratio 1 i.e. square plate, it diminishes as the aspect ratio increases beyond 
Manish Bhandari, International Journal of Emerging Trends in Engineering Research, 8(4), April 2020, 969 - 974

the value 1 . The value of non-dimensional deflection, tensile stress, shear stress, strain and shear strain for the FGM's are in between that of ceramic and metal. The values of non-dimensional results are highly grown when the FGM plate is applied with point load inspite of that the total load is kept same. This may be explained with the fact that in the case of UDL the load is uniformly distributed and whereas the point load is concentrated at a point. The strain $\left(\mathrm{e}_{\mathrm{x}}\right)$ increases with increasing value of volume fraction exponent ' $n$ '.It is also concluded that the bending response for SigmoidFGM remains smoother for various values of volume fraction index ' $\mathrm{n}$ ' as compared to that of the PowerFGM. The bending response of Exponential-FGM is quite near to the behavior of P-FGM.

The work can be expanded for variation and type of load and other ceramic metal combinations. Also thermal loading may also be imposed in addition to the mechanical loading.

\section{REFERENCES}

1. J. N. Reddy,"Analysis of functionally graded plates", International journal for numerical methods in engineering, Int. J. Numer. Meth. Engg. Vol. 47, PP 663-684, 2000.

2. Li, H; Lambros, J; Santare, M.H., "Experimental Investigation of quasi-static fracture of functionally graded materials", Int J of Solids and Structures, Vol. 37, PP. 3715-3702, 2000.

https://doi.org/10.1016/S0020-7683(99)00056-6

3. Schmauder S, Weber U, "Modeling Of Functionally Graded Materials by Numerical Homogenization", Archive of Applied Mechanics, Vol. 71, PP. 182-192, 2001. https://doi.org/10.1007/s004190000124

4. Shyang-Ho Chi, Yen-Ling Chung, "Mechanical behavior of functionally graded material plates under transverse load-Part I: Analysis", International Journal of Solids and Structures, Vol. 43, PP. 3657-3674, 2006.

https://doi.org/10.1016/j.ijsolstr.2005.04.011

5. Tahani, M; Torabizadeh, M.A.; Fereidoon, A,"Non-Linear Response of Functionally Graded Beams under Transverse Loads", 14 ${ }^{\text {th }}$ Annual (Int) Technical Engg. Conference Isfahan University of Technology Isfahan, Iran; 2006.

6. Armelle A, "Second-Order Homogenisation of Functionally Graded Materials", Int J of Solids and Structures, Vol. 47, PP. 1477-1489, 2010. https://doi.org/10.1016/j.ijsolstr.2010.02.004

7. Vanam B. C. L., Rajyalakshmi M. and Inala R., "Static analysis of an isotropic rectangular plate using finite element analysis (FEA)", Journal of Mechanical Engineering Research. Vol. 4(4), PP. 148-162, 2012.

https://doi.org/10.5897/JMER11.088

8. Jha DK, Tarun K, Singh RK."A Critical Review of Recent Research on Functionally Graded
Plates", Composite Structures, Vol. 34, PP. 458-470, 2012.

9. Yang B, Ding HJ, Chen WQ., "Elasticity Solutions For Functionally Graded Rectangular Plates With Two Opposite Edges Simply Supported", Applied Mathematical Modeling, Vol. 36, PP. 488-503, 2013. https://doi.org/10.1016/j.apm.2011.07.020

10. Bhandari, M. Purohit, K., "Static Response of Functionally Graded Material Plate under Transverse Load for Varying Aspect Ratio", International Journal of Metals. 2014. http://dx.doi.org/10.1155/2014/980563. 11. Moita, J.; Araújo, A; Correia, V.; Soares, C.; Herskovits, J., "Buckling and nonlinear response of functionally graded plates under thermo-mechanical loading", Composite Structures, Vol. 202, PP. 719-730, 2018.

https://doi.org/10.1016/j.compstruct.2018.03.082

12. Yogesh P. and Atul B., "Performance evaluation of the Indian plastic processors supply chain:Implementing lean and green philosophies", IJETER, Vol.7:5, PP. 1-14. https://doi.org/10.30534/ijeter/2019/01752019

13. Kuntal $\mathrm{M}$ and Padamati S., "Wild detection and Recognition from aerial videos using computer vision techniques", IJETER, Vol.7:5, PP. 15-21.

14. Edward B., "Microcontroller-based wearable blood pressure monitoring device with GPS and SMS feature through mobile app." IJETER,Vol.7:6, PP. 3235 .

https://doi.org/10.30534/ijeter/2019/02762019 